\title{
Fratura complexa do osso zigomático: relato de caso
}

\author{
Zygomaticomaxillary complex fracture: case report \\ Fractura de hueso cigomático: reporte de un caso \\ Rodrigo dos Santos PEREIRA ${ }^{1}$ \\ Jonathan Ribeiro da SILVA ${ }^{2}$ \\ Paulo Roberto BARBOSA JÚNIOR ${ }^{3}$ \\ Luís Fernando MAGACHO ${ }^{4}$ \\ Gustavo Lima LATINI ${ }^{5}$ \\ ${ }^{I}$ Staff do Serviço de Cirurgia e Traumatologia do Hospital Geral de Nova Iguaçu - RJ, Brasil \\ Especialista em Cirurgia e Traumatologia Bucomaxilofacial \\ Mestre e Doutor em Odontologia pela Faculdade de Odontologia de Araçatuba, Universidade Estadual Paulista,UNESP \\ Fellow AO pela Universitätsklinikum Eppendorf. Hamburgo, Alemanha \\ ${ }^{2}$ Especialista em Cirurgia e Traumatologia Bucomaxilofacial, Mestre em Odontologia pela UNIGRANRIO \\ Doutorando em Odontologia pela Faculdade de Odontologia de Araçatuba - UNESP \\ ${ }^{3}$ Especialista em Cirurgia e Traumatologia Bucomaxilofacial, Mestre em Odontologia pela São Leopoldo Mandic. \\ Staff do Serviço de Cirurgia e Traumatologia do Hospital Geral de Nova Iguaçu - RJ, Brasil \\ ${ }^{4}$ Especialista em Cirurgia e Traumatologia Bucomaxilofacial, Mestre em Odontologia pela UNIGRANRIO \\ Staff do Serviço de Cirurgia e Traumatologia do Hospital Geral de Nova Iguaçu - RJ, Brasil \\ ${ }^{5}$ Especialista em Cirurgia e Traumatologia Bucomaxilofacial, \\ Chefe do Serviço de Cirurgia e Traumatologia do Hospital Geral de Nova Iguaçu - RJ, Brasil
}

\begin{abstract}
Resumo
O tratamento das fraturas complexas do osso zigomático representa um desafio para o cirurgião bucomaxilofacial, pois a presença de deslocamento do corpo do zigomático e cominuição das paredes orbitárias podem gerar sequelas significativas ao paciente. O princípio básico para o manejo destas fraturas está na adequada exposição e redução dos segmentos fraturados para o restabelecimento das dimensões faciais pré-trauma. O objetivo deste trabalho é relatar um caso clínico de paciente vítima de fratura complexa zigomaticomaxilar, tratada com fixação interna estável com placas e parafusos do sistema 1.5 e $2.0 \mathrm{~mm}$. O paciente foi submetido à cirurgia de redução e fixação da fratura via acesso coronal, intrabucal, e subciliar, e, após o período de 02 meses de acompanhamento pós-operatório, pode retornar ao convívio social sem sequelas.
\end{abstract}

Descritores: Zigoma; Fraturas Orbitárias; Fixação Interna de Fraturas.

\begin{abstract}
The treatment of the zygomatic fractures presents a challenge for the maxillofacial surgeon, because the presence of displacement of the zygomatic body and comminution of the orbital walls can generate significant sequelae to the patient. The basic principle for the management of these fractures is the adequate exposure and reduction of the fractured segments, for the reestablishment of the pre-trauma facial dimensions. The objective of this study is to report a clinical case of a patient suffering from a complex zygomaticmaxillary fracture treated with stable internal fixation with plates and screws of 1.5 and $2.0 \mathrm{~mm}$ system. The patient was submitted to open reduction and internal fixation s through coronal, intra-buccal, and subtarsal access, and after a period of 2 months postoperative follow-up, she could return to social life without sequelae.

Descriptors: Zygoma; Orbital Fractures; Fracture Fixation, Internal.

Resumen

El tratamiento de las fracturas complejas del hueso cigomático presenta un reto para el cirujano maxilofacial, ya que la presencia del desplazamiento del cuerpo cigomático y trituración de las paredes orbitarias, puede generar consecuencias importantes para el paciente. El principio básico para el tratamiento de estas fracturas se encuentra en la exposición adecuada y la reducción de segmentos fracturados, para la restauración de las dimensiones faciales pretrauma. El objetivo de este trabajo es presentar un caso de un paciente con fractura del complejo cigomático-maxilar tratado con fijación interna estable con mini placa y tornillos del sistema 1.5 y 2,0 $\mathrm{mm}$. La paciente fue sometida a una cirugía de bypass y la fijación de la fractura a través del acceso coronal, intraoral, y subciliar, y después del período de 02 meses de seguimiento postoperatorio puede volver a la vida social sin secuelas.

Descriptores: Cigoma; Fracturas Orbitales; Fijación Interna de Fracturas.
\end{abstract}

\section{INTRODUÇÃO}

O complexo zigomaticomaxilar (CZM) é uma das estruturas mais proeminentes da face, e por presentar essa particularidade torna-se vulnerável, estando frequentemente envolvido no trauma facial ${ }^{1}$. A análise epidemiológica destas fraturas é dependente da condição socioeconômica, cultural, e geográfica do local de estudo, sendo que a maior parte dos trabalhos encontram um predomínio de pacientes do gênero masculino, de $21-40$ anos de idade ${ }^{2,3}$.

Quando o CZM sofre um trauma de alta energia, a moldura orbital e as paredes internas da órbita podem sofrer fraturas com alta complexidade, apresentado deslocamentos e cominuição que dificultam o tratamento cirúrgico ${ }^{4}$. A abordagem destas fraturas é baseada no acesso direto a todos os pontos fraturados via acessos cirúrgicos amplos, redução óssea com reestabelecimento das dimensões da face, fixação de no mínimo 03 pontos com placas e parafusos dos sistemas 1.5 e $2.0 \mathrm{~mm}$, seguido de reconstrução das paredes internas orbitárias utilizando-se enxertos autógeno, malhas de titânio ou biomateriais ${ }^{5,6}$.

As complicações pós-operatórias associadas a estas fraturas são a diplopia, distopia, enoftalmia e perda de projeção do osso malar ${ }^{7}$. A falta de uma adequada redução anatômica na moldura orbitária, e um aumento do volume orbital por deficiência na reconstrução de suas paredes, são os principais motivos para sequelas e complicações do pósoperatório de $\mathrm{CZM}^{7,8}$. 
O objetivo deste trabalho é relatar um caso clínico de paciente vítima de fratura complexa zigomaticomaxilar, tratada com fixação interna funcionalmente estável.

\section{CASO CLÍNICO}

Paciente, gênero feminino, 25 anos de idade compareceu à emergência do Hospital Geral de Nova Iguaçu vítima de colisão entre motocicletas. Ao exame clínico apresentava ferimento corto contuso em região palpebral superior e superciliar direita. A palpação apresentava crepitação óssea na região da crista zigomático-maxilar e margem infraorbital do lado direito. Relatava queixas álgicas na região malar direita além de diplopia e distopia a direita, porém sem oftalmoplegia e enoftalmo. A paciente foi submetida à tomografia computadorizada onde se observou fratura complexa do osso zigomático direito com segmentação óssea na margem infraorbital, sutura frontozigomática (Figura 1), no arco zigomático (Figura 2) e do teto e soalho orbitário (Figura 3). Além disso, ocorreu fratura do processo coronoide direito e da maxila direita. Os ferimentos mencionados foram suturados e a paciente foi internada, sendo solicitados exames pré-operatórios para a realização do procedimento cirúrgico eletivo.

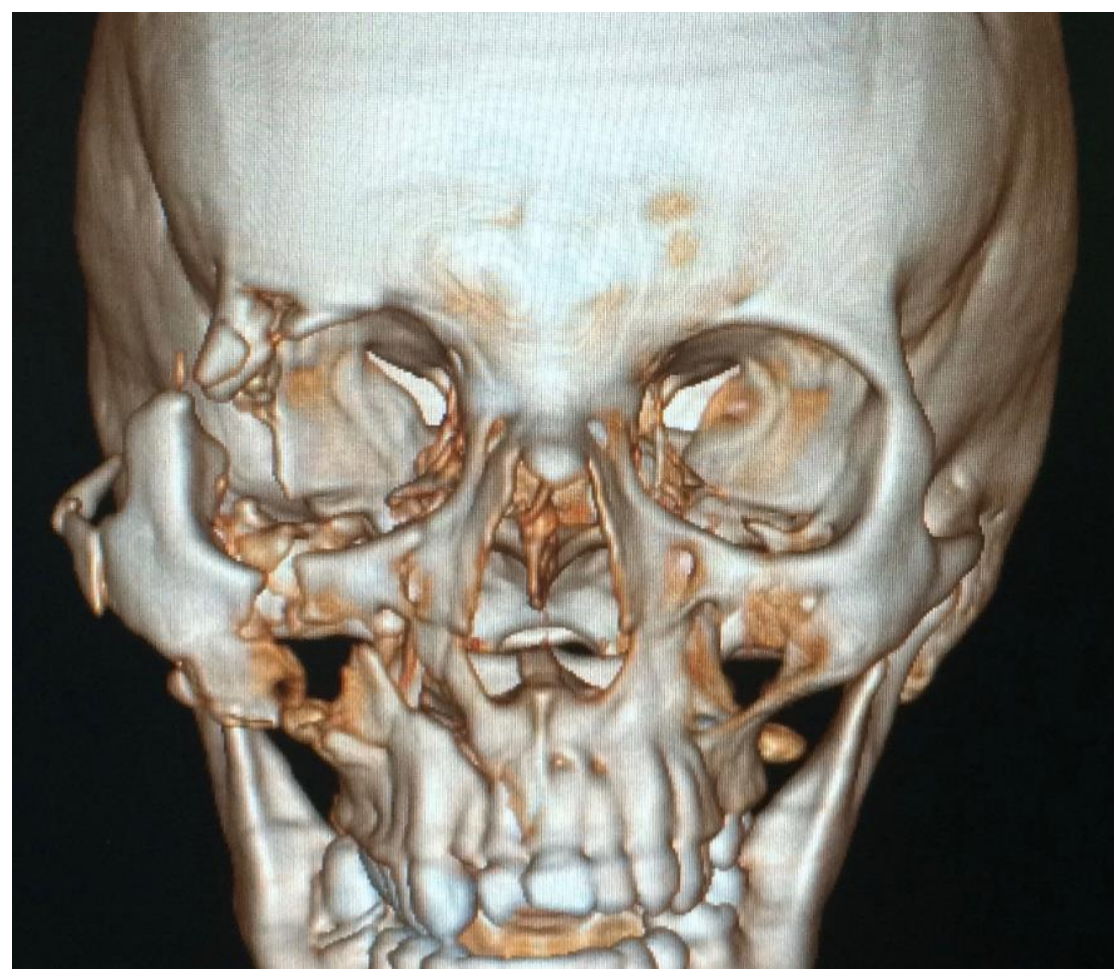

Figura 1: Reconstrução 3D da tomografia computadorizada, na vista frontal, evidenciando as fraturas do osso zigomático e da maxila direita.

Após 3 dias, a paciente foi encaminhada ao centro cirúrgico e anestesiada sob intubação orotraqueal. Um acesso coronal com extensão pré-auricular a direita foi feito para expor as fraturas do arco zigomático, sutura frontozigomática e corpo do osso zigomático; o acesso subciliar foi realizado para expor a margem infraorbital e o soalho orbitário; e o acesso intraoral vestibular para expor as fraturas na crista zigomático-maxilar. Um parafuso $1.5 \mathrm{~mm}$ foi instalado no corpo do osso zigomático para auxiliar na redução e correto posicionamento da sutura esfenozigomático e garantir a projeção anteroposterior do osso. O primeiro ponto fixado foi a sutura frontozigomática onde uma placa em "L" do sistema $1.5 \mathrm{~mm}$ foi utilizada para simplificar a fratura e em seguida uma placa do sistema $2.0 \mathrm{~mm}$ fixada com 2 parafusos no osso frontal e 2 no próprio osso zigomático (Figura 4). Em sequência, o segundo ponto a ser fixado foi na região da crista zigomático-maxilar onde uma placa reta longa do sistema $2.0 \mathrm{~mm}$ foi fixada no corpo do malar e na maxila direita devido a cominuição da crista no trauma. A margem infraorbital foi fixada em duas partes em decorrência do segmento ósseo intermediário. A primeira fixação foi com uma placa em "L" $1.5 \mathrm{~mm}$ unindo o segmento ao processo frontal da maxila e depois uma placa reta unindo o segmento ao osso zigomático (Figura 5 ). O arco zigomático teve seus segmentos ósseos fixados com 2 placas retas do sistema $1.5 \mathrm{~mm}$, o pilar canino com 1 placa e a parede anterior do seio maxilar direito com uma placa em "L" (Figura 6).

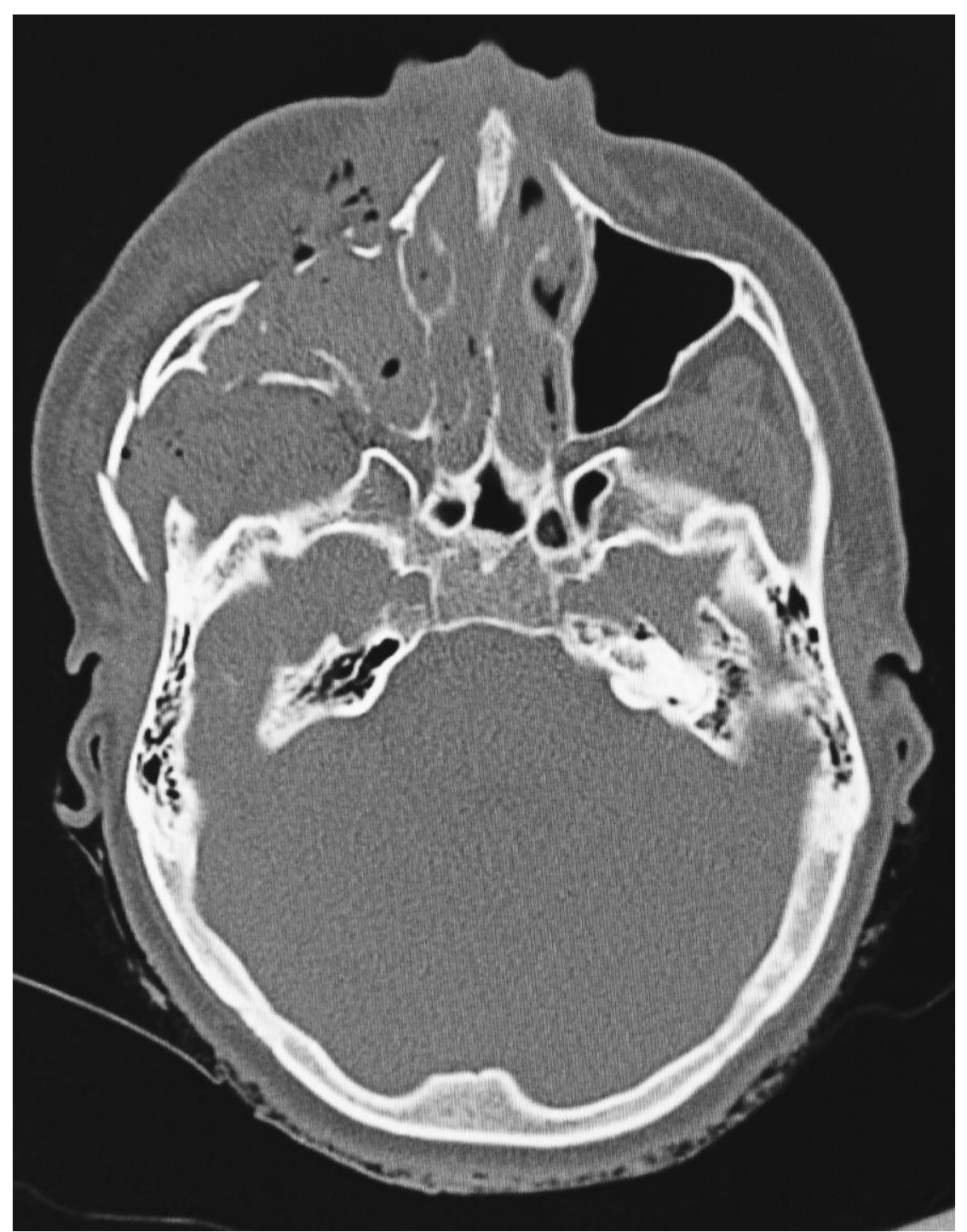

Figura 2: Corte axial da tomografia computadorizada mostrando as fraturas na maxila e arco zigomático.

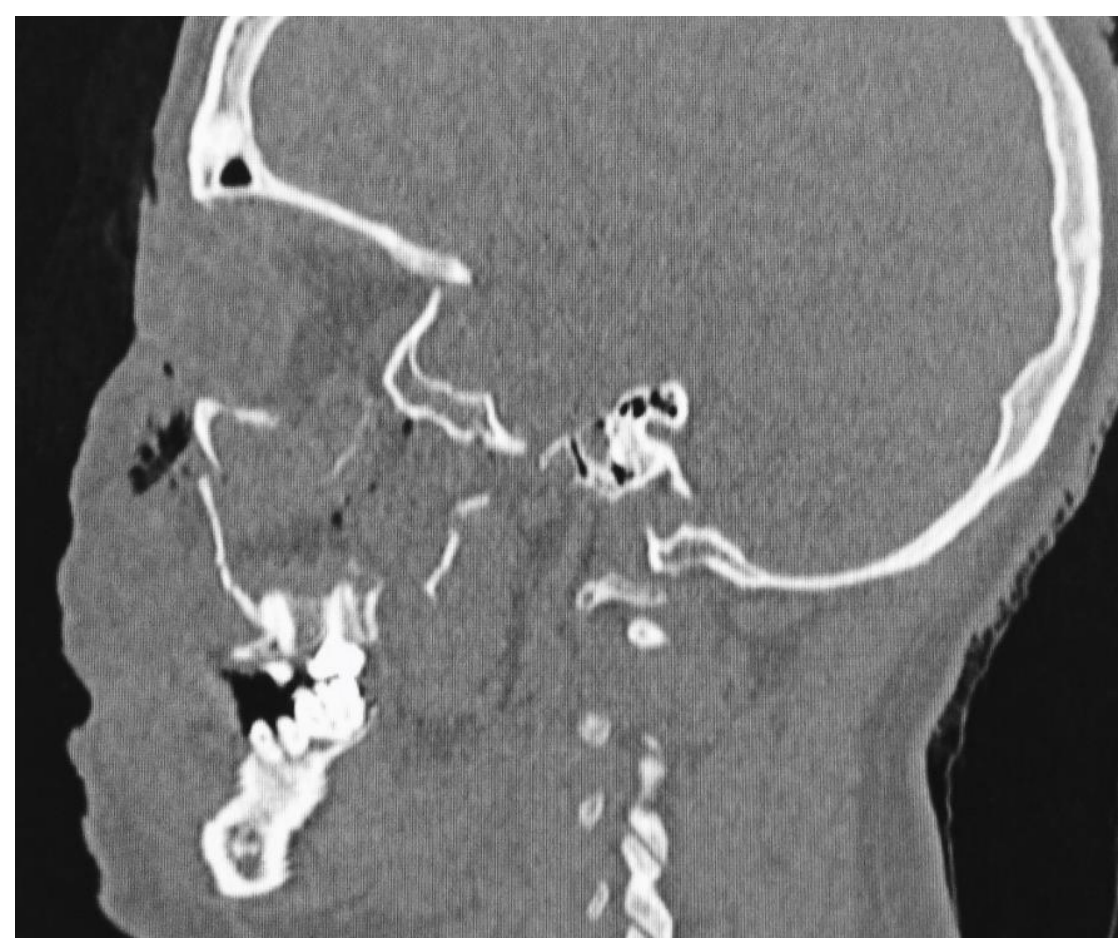

Figura 3: Imagem em corte sagital evidenciando a fratura no soalho orbital direito.

$\mathrm{O}$ teto da órbita, assim como o soalho foram restaurados com uma malha de titânio $1.5 \mathrm{~mm} \mathrm{e}$, posteriormente, realizado o teste de ducção forçada para 
verificação de aprisionamento de algum músculo extrínseco do olho.

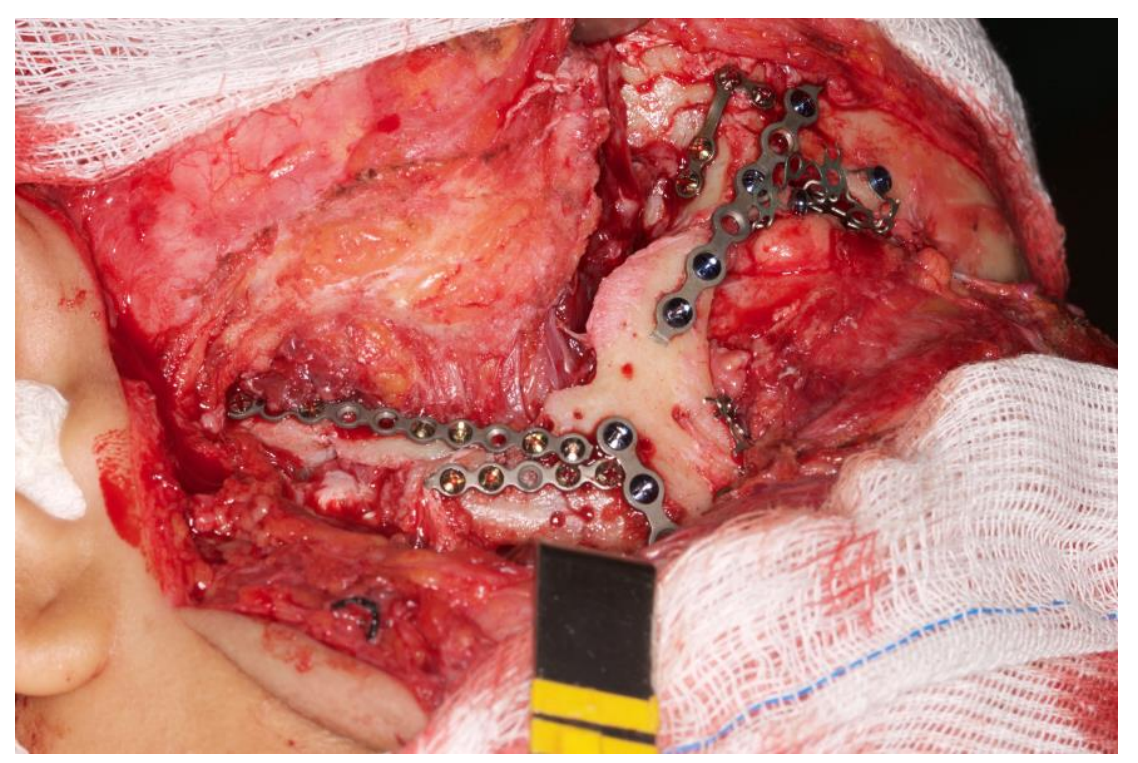

Figura 4: Imagem transcirúrgica demonstrando o arco zigomático fixado com 2 placas retas do sistema $1.5 \mathrm{~mm}$; uma placa em "L" 1.5 mm utilizada para simplificar o segmento ósseo na sutura frontozigomática com uma placa reta $2.0 \mathrm{~mm}$ fixando a fratura; observa-se, também, a tela de titânio no teto orbitário.

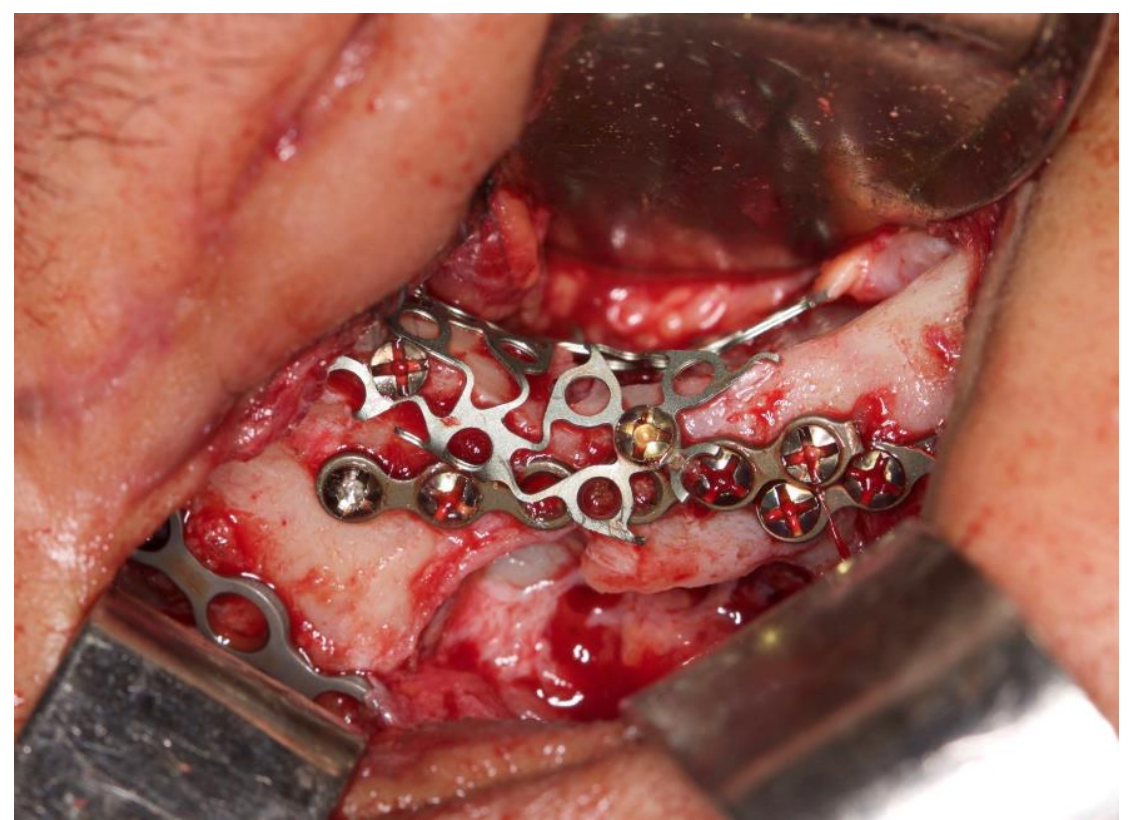

Figura 5: Imagem transcirúrgica demonstrando a fixação da margem infraorbitária com placas do sistema $1.5 \mathrm{~mm}$ e a tela para reconstrução do soalho orbitário.

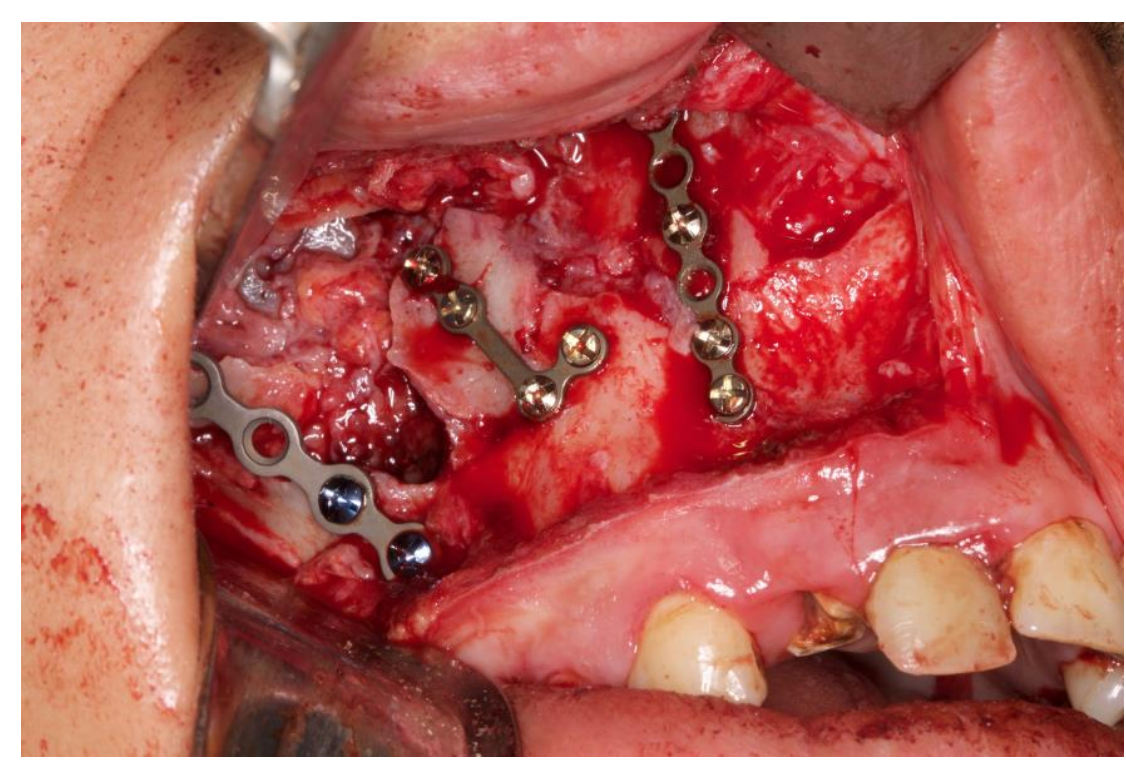

Figura 6: Imagem transcirúrgica intraoral evidenciando a fixação da crista zigomaticomaxilar, parede anterior do seio maxilar e pilar canino.

A fratura do processo coronoide não foi abordada, optando-se por trata-la conservadoramente. A paciente permaneceu no hospital por 24 horas, sob observação, onde não foi relatada nenhuma complicação tendo assim alta hospitalar com as orientações pós-operatórias. A mesma foi acompanhada semanalmente no ambulatório e, após 2 meses, não foi evidenciada nenhuma complicação com o retorno das funções mastigatórias e a recuperação das queixas oftalmológicas (Figuras 7 e 8).

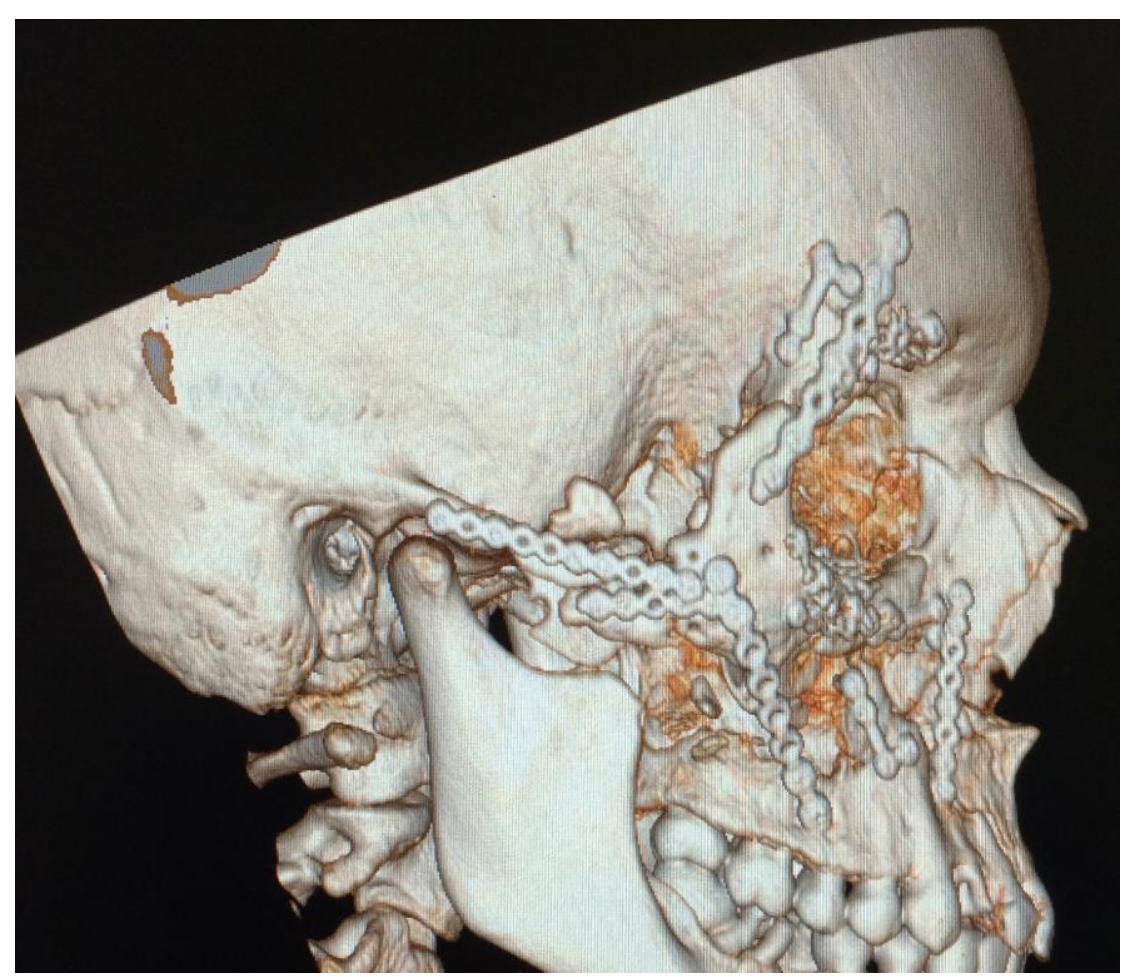

Figura 7: Reconstrução 3D da tomografia computadorizada, em uma vista lateral, do pós operatório de 2 meses evidenciando as fixações instaladas.

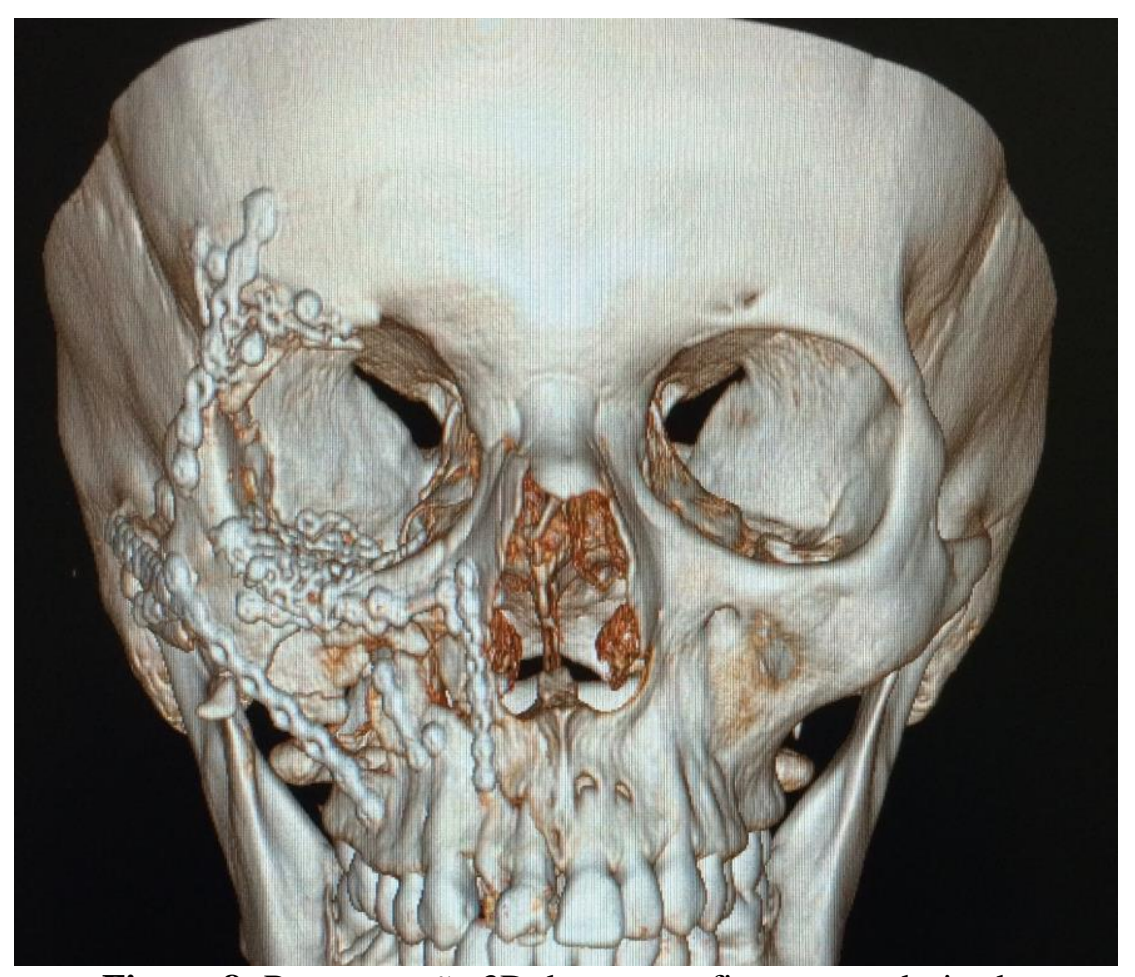

Figura 8: Reconstrução 3D da tomografia computadorizada, em uma vista frontal, do pós operatório de 2 meses evidenciando as fixações instaladas.

\section{DISCUSSÃO}

O osso zigomático têm um importante papel em 3 fatores na face: na estética, pois estabelece o contorno facial e a projeção anteroposterior do terço médio da face; proteção, pois apresenta-se em uma posição chave para absorção de impactos de estruturas importantes como o cérebro e a cavidade orbitária; e por último a função, pois fraturas deste complexo ocasionam problemas nas funções oculares, do nervo infraorbital e também na abertura bucal ${ }^{9}$.

As fraturas complexas do osso zigomático representam um desafio para qualquer cirurgião maxilofacial. O grande deslocamento das paredes e molduras orbitárias dificulta a redução e aumentam o risco de deformidades e sequelas pós-cirúrgicas ${ }^{7,8}$. A redução aberta e fixação interna estável representa o método mais 
indicado para essas fraturas, por apresentar maior estabilidade e melhores resultados, porém não existe consenso no número e posição das placas para fixação ${ }^{10}$.

Em casos de cominuição, como o relatado neste artigo, é consenso na literatura que uma ampla exposição das fraturas se torna necessária ${ }^{11}$. Além disso, a correta sequencia de fixação e o reparo dos pontos anatômicos, tais como o arco zigomático e principalmente a sutura esfenozigomatica, permitem o posicionamento tridimensional do osso zigomático. Quando o cirurgião tende a ser conservador, a visualização e o alinhamento do arco zigomático e dos componentes orbitários não providenciam informações suficientes para a redução e projeção anteroposterior.

Segundo Barry et al. ${ }^{12}$ a sequência de fixação deve seguir primeiramente a estabilização dos pilares verticais como a sutura frontozigomatica e a crista zigomaticomaxilar. Contudo, a literatura não é concisa quanto ao qual dos dois deve ser primeiramente estabilizado $^{13-15}$. No presente caso, optamos por fixar a margem frontozigomática a princípio, pois como o arco zigomático encontrava-se segmentado, o único ponto de referência da projeção do osso zigomático era a sutura esfenozigomática.

Chakranarayan et al. ${ }^{4}$ realizaram um estudo avaliando a eficácia de fixação das fraturas zigomáticos em 02 pontos, e concluíram que a fixação da região frontozigomática e do pilar zigomaticomaxilar são satisfatórias como método de fixação para as fraturas do osso zigomático.

Kim et al. ${ }^{16}$, em 2011, compararam a fixação de 01 ponto com 02 pontos nas fraturas zigomáticas e obtiveram, como resultado, uma adequada fixação quando aplicado 01 ponto de fixação em casos selecionados e de baixa complexidade, diferentemente do apresentado neste caso.

$\mathrm{O}$ menor número de pontos de fixação diminui a possibilidade de cicatriz facial, morbidade pós-operatória, e o custo da cirurgia ${ }^{16}$. Porém, em casos de fraturas complexas, fica impossível a manutenção da redução anatômica com apenas 01 ponto de fixação. Dependendo do grau de deslocamento, um terceiro ponto precisa ser fixado para prevenir sequelas pós operatórias ${ }^{17}$.

\section{CONCLUSÃO}

Neste artigo demonstra-se a sequência e os pontos corretos para a redução e fixação em um caso de fratura complexa do osso zigomático e da maxila. Um caso de alto grau de complexidade ocasionado por um trauma direto e de alta energia. Após 2 meses de pós-operatório, não houve sequelas e a paciente retornou às suas funções mastigatórias e oftalmológicas.

\section{REFERÊNCIAS}

1. Rosado P, de Vicente JC. Retrospective analysis of 314 orbital fractures. Oral Surg Oral Med Oral Pathol Oral Radiol. 2012; 113(2):168-71.

2. Gomes PP, Passeri LA, Barbosa JR. A 5-year retrospective study of zygomatico-orbital complex and zygomatic arch fractures in Sao Paulo State, Brazil. J Oral Maxillofac Surg. 2006; 64(1):63-7.

3. Trivellato PF, Arnez MF, Sverzut CE, Trivellato AE. A retrospective study of zygomatico-orbital complex and/or zygomatic arch fractures over a 71-month period. Dent Traumatol. 2011; 27(2):135-42.

4. Chakranarayan A, Thapliyal GK, Sinha R, Suresh MP. Efficacy of two point rigid internal fixation in the management of zygomatic complex fracture. J Maxillofac Oral Surg. 2009; 8(3):265-9.

5. Yang L, Liu C, Hua W, Liu W, Wang X, Wang K, et al. Sequential reduction and fixation for zygomatic complex fractures. Zhongguo Xiu Fu Chong Jian Wai Ke Za Zhi. 2013; 27:1181-4.

6. Olate S, Lima SM, Jr, Sawazaki R, Moreira RW, de Moraes M. Surgical approaches and fixation patterns in zygomatic complex fractures. J Craniofac Surg. 2010; 21(4):1213-7.

7. Kristensen S, Tveteras K. Zygomatic fractures: classification and complications. Clin Otolaryngol Allied Sci. 1986; 11(3):123-9.

8. Giudice M, Colella G, Marra A. The complications and outcomes of fractures of the orbital-maxillary-zygomatic complex. Minerva Stomatol. 1994; 43(1-2):37-41.

9. Marinho RO, Freire-Maia B. Management of fractures of the zygomaticomaxillary complex. Oral Maxillofac Surg Clin North Am. 2013; 25(4):617-36.

10. Calderoni DR, Guidi Mde C, Kharmandayan P, Nunes $\mathrm{PH}$. Seven-year institutional experience in the surgical treatment of orbito-zygomatic fractures. J Craniomaxillofac Surg. 2011;39(8):593-9.

11. Hollier LH, Thornton J, Pazmino P, Stal S. The management of orbitozygomatic fractures. Plast Reconstr Surg. 2003; 111(7):2386-92.

12. Barry CP, Ryan WJ, Stassen LF. Anatomical study of factors contributing to zygomatic complex fracture instability in human cadavers. Plast Reconstr Surg. 2007; 119(2):637-40.

13. Habal MB. The orbits: it is less important what you put in than how you secure it. J Craniofac Surg. 2010; 21(4):965-6

14. Yamashita M, Kishibe M, Shimada K. Incidence of lower eyelid complications after a transconjunctival approach: influence of repeated incisions. J Craniofac Surg. 2014; 25:1183-6.

15. Ellis E 3rd, Kittidumkerng W. Analysis of treatment for isolated zygomaticomaxillary complex fractures. J Oral Maxillofac Surg. 1996; 54(4):386-400.

16. Kim ST, Go DH, Jung JH, Cha HE, Woo JH, Kang IG. Comparison of 1-point fixation with 2-point fixation in treating tripod fractures of the zygoma. J Oral Maxillofac Surg. 2011; 69(11):2848-52.

17. Rana M, Warraich R, Tahir S, Igbal A, von See C, EcKardt AM, et al. Surgical treatment of zygomatic bone fracture using two points fixation versus three point fixation--a randomised prospective clinical trial. Trials. 2012; 12:13:36

\section{CONFLITO DE INTERESSES}

Os autores declaram não haver conflitos de interesse.

\section{AUTOR PARA CORRESPONDÊNCIA}

\section{Rodrigo dos Santos Pereira}

dr.pereira@live.com

Submetido em 03/01/2017 Aceito em 10/03/2017 